\title{
Simple quantum circuit for pattern recognition based on nearest mean classifier
}

\author{
Gharib S. Mahmoud ${ }^{1,2}$, Azeddine Messikh ${ }^{3}$ \\ ${ }^{1}$ Dept. of Science in Engineering, Kulliyah of Engineering, IIUM, Kuala Lumpur, Malaysia. \\ ${ }^{2}$ Faculty of Industrial Education, Science Department, Beni-Suef University, Egypt. \\ ${ }^{3}$ Dept. of Computer Science, Kulliyah of Information and communication technology, \\ IIUM, Kuala Lumpur, Malaysia.
}

\begin{abstract}
Machine learning plays a key role in many applications such as data mining and image recognition. Classification is one subcategory under machine learning. In this paper we propose a simple quantum circuit based on the nearest mean classifier to classified handwriting characters. Our circuit is a simplified circuit from the quantum support vector machine [Phys. Rev. Lett. 114, 140504 (2015)] which uses quantum matrix inverse algorithm to find optimal hyperplane that separated two different classes. In our case the hyperplane is found using projections and rotations on the Bloch sphere.
\end{abstract}

Keywords- Quantum circuit and Pattern recognition.

\section{INTRODUCTION}

Quantum mechanics is emerging in many disciplines especially in computer science as natural evolution. One of the most important goals in computer science, especially in pattern recognition, is to build a quantum machine learning $[1-3 \mid$ that will be able to classify objects in different classes. This is because it has been demonstrated that quantum algorithms can improve the complexity of the classical algorithms and also can be superior to their classical counterparts in term of reducing resource costs. The nearest-mean algorithm [4] is one of the simplest and accurate algorithms that has been used in handwriting recognition and as well as in astronomy [5,6].

In pattern recognition $[7,8]$ there are two major types of classical machine learning [9-11], the supervised learning and the unsupervised learning. Supervised learning is the type of machine learning where the training data are labeled in advance, whereas the unsupervised learning the data are not labeled. The support vector machine (SVM) is an example of supervised model, and it is a decision machine that is used for classification, regression and detection [9].

The SVM belongs to quadratic programming problems, which can be solved classically in time proportional to $O(\operatorname{poly}(N, M))$, where $N$ is the dimension of the feature space and $M$ is the number of training vectors. Whereas, quantum support vector machine (QSVM) introduced by Rebentrost et al. [12] can be implemented with only $O(\log N M)$. This is due to the fact that QSVM is based on the solution of linear system of equations which can be obtained using quantum algorithm more efficient than any classical algorithm [13-15]. Furthermore, the quantum algorithm for solving linear system of equations is based on quantum matrix inversion algorithm and it is already available [13]. Recently, an experimental realization of quantum algorithm for solving linear systems of equations $2 \times 2$ has been reported [16]. It is implemented by using four-qubit nuclear magnetic resonance (NMR) processor and the fidelities obtained are all above $96 \%$. The setup of this experimental has been adapted to implement quantum support vector machine using the same NMR processor [17].

In this paper we propose a simple quantum circuit based on pattern recognition on the quantum Bloch sphere and the nearest mean classifier (NMC) [18]. Moreover, this quantum circuit does not require quantum matrix inversion and can be implemented by using up to three-qubit NMR processor. In this case the hyperplane which separetes the classes can be found using projections and rotations on the Bloch sphere.

The paper is organized as follows. Section II reviews the quantum support vector machine which is based on quantum inverse matrix algorithm [17], and the pattern recognition on Bloch sphere proposed by Sergioli et al. [19]. In section III we propose a simple quantum circuit and at the end we give a conclusion. 


\section{BACKGROUND}

\section{Quantum support vector machine circuit}

The support vector machine algorithm outputs a separating hyperplane from given labeled training data (supervised learning). It is based on linear equations which can be put in the form $A \vec{x}=\vec{b}$, where $A$ is a Hermitian matrix. The solution of such system of equations can be obtained using quantum algorithm as shown in [13,16]. Let briefly review this algorithm.

The spectral decomposition of the Hermitian matrix $A$ is given by $A=\sum_{i} \lambda_{i}\left|u_{i}\right\rangle\left\langle u_{i}\right|$, where $\lambda_{i}$ are the eigenvalues of $A$ and $\left|u_{i}\right\rangle$ are the corresponding eigenvectors. By writing $|b\rangle=\beta_{i}\left|u_{i}\right\rangle$, the solution is obtained using the inverse of the matrix $A$, i.e., $|x\rangle \propto A^{-1}|b\rangle=\sum_{i}\left(\beta_{i} / \lambda_{i}\right)\left|u_{i}\right\rangle$. A quantum circuit for solving a system of two equations with two variables has been recently implemented using a four-qubit NMR quantum information processor [16]. It has three main subcircuits: phase estimation, rotations with angles $\lambda_{i}^{-1}$, and inverse phase estimation.

The quantum support vector machine has been also realized experimentally using four-qubit processor [17]. The classification is obtained by measuring the expected value of a coherent term [17]. The quantum circuit for this experiment consists of three main parts. The first part is a quantum circuit that solve the linear set of equations using the quantum matrix inversion. The matrix $A$ is a $2 \times 2$ Hermitian matrix which has been chosen such that the two eigenvalues are in the form $2^{i}$, i.e., 1 and 2 , and the vector $\vec{b}$ is set to $|b\rangle=\frac{1}{\sqrt{2}}(|0\rangle+|1\rangle)$. The solution of the set of linear equations is used to obtain the hyperplane parameters. The second part of the quantum circuit is a training-data oracle which is implemented by two controlled rotation gates with angles depending on the training data. It is used to realize the kernel matrix (up to a constant factor) which is crucial in SVM. The third one introduces the query vector and it is used for classification. The experiment realization of QSVM has been implemented to classify handwritten characters " 6 " and "9" [17].

We have seen in this section that the quantum circuit for solving a system of linear equations is used to classify a query using QSVM. In the next section we will show that we can simplify the circuit by using the idea of pattern-recognition in Bloch sphere with the nearest mean classifier which is the simplest accurate algorithm for classification.

\section{Pattern recognition on Bloch sphere}

It is well known that any qubit $|\psi\rangle$ can be represented as a point on the surface of the Bloch sphere. The corresponding density matrix of this qubit $\rho=|\psi\rangle\langle\psi|$ can be written in the general form

$$
\rho=\frac{1}{2}(I+\vec{n} \cdot \vec{\sigma}),
$$

where $\vec{n}$ is a unit vector and $\vec{\sigma}=\left(\sigma_{x}, \sigma_{y}, \sigma_{z}\right)$, the well known Pauli matrices.

Using stereographic projection [20], each point $\left(x_{1}, x_{2}\right)$ in two-dimentional space can be mapped to a point $\left(r_{1}, r_{2}, r_{3}\right)$ on the surface of the Bloch sphere. This is very important in two-feature patterns recognition. Recently, G Segioli et al. have investigated pattern recognition on the quantum Bloch sphere [19]. They have shown that there is one-to-one correspondence between two-feature patterns and density matrices. Here we briefly review their idea.

Let a training data consists of two classes $A$ and $B$. Each class contains points in two-dimentional space. First, let consider the map

$$
\begin{aligned}
\left(x_{1}, x_{2}\right) & \longrightarrow\left(r_{1}, r_{2}, r_{3}\right) \\
& =\left(\frac{2 x_{1}}{x_{1}^{2}+x_{2}^{2}+1}, \frac{2 x_{2}}{x_{1}^{2}+x_{2}^{2}+1}, \frac{x_{1}^{2}+x_{2}^{2}-1}{x_{1}^{2}+x_{2}^{2}+1}\right),
\end{aligned}
$$

This map is the stereographic projection of the point $\left(x_{1}, x_{2}\right)$ on the Bloch sphere. It is clear that this projection is a one-to-one correspondence between the twofeature patterns and the points on the Bloch sphere. That is,

$$
\left(x_{1}, x_{2}\right) \longrightarrow|\psi\rangle=\frac{|x|}{\sqrt{1+|x|^{2}}}|0\rangle+\frac{\mathrm{e}^{i \phi}}{\sqrt{1+|x|^{2}}}|1\rangle,
$$

where $|x|$ is the magnitude of the complex number $x=x_{1}+i x_{2}$ and $\phi$ its phase. Writing Eq. (2) in the form of density matrix

$$
\left(x_{1}, x_{2}\right) \longrightarrow \rho=\frac{1}{|x|^{2}+1}\left(\begin{array}{cc}
|x|^{2} & |x| \mathrm{e}^{-i \phi} \\
|x| \mathrm{e}^{i \phi} & 1
\end{array}\right) .
$$

yields to a one-to-one correspondence between the twofeature patterns and the density matrices. Second, in order to use the density matrices in pattern recognition, a suitable definition of distance is needed. This can be performed by using a normalized trace distance between density operators [19] which measures the distance between two density matrices $\rho_{a}$ and $\rho_{b}$. This distance is defined as follows.

$$
d\left(\rho_{a}, \rho_{b}\right)=K \sum_{i}\left|\lambda_{i}\right|
$$


where $\lambda_{i}$ are the eigenvalues of the matrix $\rho_{a}-\rho_{b}$ and $K$ is a normalize factor which is given by $K=$ $\frac{1}{\sqrt{\left(1-r_{a 3}\right)\left(1-r_{b 3}\right)}}$, with $r_{a 3}$ and $r_{b 3}$ are the $\sigma_{z}$ Pauli components of $\rho_{a}$ and $\rho_{b}$, respectively. It is worth mentioning that this distance given by Eq. (4) preserves the order, i.e., given three points $x_{a}, x_{b}$ and $x_{c}$ such that the distances satisfying $d\left(x_{a}, x_{b}\right) \leq d\left(x_{b}, x_{c}\right)$, then the corresponding density matrices satisfy a similar inequality, $d\left(\rho_{a}, \rho_{b}\right) \leq d\left(\rho_{b}, \rho_{c}\right)$. Third, in order to classify an unknown two-feature pattern, a minimum distance classifier can be used. It is also called the Nearest Mean Classifier (NMC) [21]. This is the simplest classification algorithm that can be used to classify a query into lineary separated classes. Finally, It was found that the quantum discriminant function based on NMC is given by [19]

$$
f(\vec{r})=\mathbf{F} . \mathbf{r}+\tilde{K}^{2}-1=0,
$$

where

$$
\begin{aligned}
\mathbf{F} & =\left(r_{a_{1}^{*}}-\tilde{K}^{2} r_{b_{1}^{*}}, r_{a_{2}^{*}}-\tilde{K}^{2} r_{b_{2}^{*}}, r_{a_{2}^{*}}-\tilde{K}^{2} r_{b_{3}^{*}}\right), \\
\tilde{K} & =\sqrt{\frac{1-r_{a_{3}^{*}}}{1-r_{b_{3}^{*}}}}
\end{aligned}
$$

where $r_{i^{*}}=\left(r_{i_{1}^{*}}, r_{i_{2}^{*}}, r_{i_{3}^{*}}\right)$ is the estimated centroid point of the class $i$ computed from the training data. If $f(r)>0$ the unknown two-feature pattern belongs to the class $A$, otherwise it belongs to the class $B$. The quantum discriminant function (5) is a set of linear equations which can be easily solved. We will show in the next section how to obtain its solution without the need of quantum inverse matrix algorithm.

\section{Quantum Circuit Based on NMC}

In order to use the idea in Sec. II for pattern recognition, we need to find an approach to reduce the image to a vector of two-dimension, $\vec{x}=\left(x_{1}, x_{2}\right)$. Zhaokai Li et al. [17] have used a very simple approach to do so. They used the vertical and horizontal ratios. This will reduce all the images to vectors of two-dimension. We will adopt their approach. If the two classes are well linearly separated one can use NMC to classify them.

As an example, we plot in Fig. 1 the two-feature patterns for the handwriting characters " 6 " and " 9 " taken from Ref. [22]. One can see that the horizontal ratio and the vertical ratio make the two characters linearly separated.

Support Vector Machine is a classifier that classifies a query $\vec{r}_{0}$ into one of two classes, $f_{\text {svm }}\left(\vec{r}_{0}\right)= \pm 1$, depending on the class to which the query belongs.
This means that the classifier finds an optimal hyperplane $\vec{w} \cdot \vec{r}+b=0$ that divides the two classes. The classification of a query $\vec{r}_{0}$ is giving by

$$
\begin{aligned}
f_{\text {svm }}\left(\vec{r}_{0}\right) & =\operatorname{sgn}\left(\vec{w} \cdot \vec{r}_{0}+b\right), \\
& =\operatorname{sgn}\left(\sum \alpha_{i}\left(\vec{r}_{i} \cdot \vec{r}_{0}\right)+b\right),
\end{aligned}
$$

where the normal vector $\vec{w}=\sum \alpha_{i} \vec{r}_{i}$. The role of SVM is to determine the values of $\alpha_{i}$. Patrick et. al. [12] implemented the support vector machine quantum mechanically using quantum inverse matrix algorithm to determine the values of $\alpha_{i}$. We can pass around the use of the quantum inverse matrix algorithm by using NMC to determine the values of $\alpha_{i}$. This is an important simplification of the quantum circuit proposed in Sec. I] (see Ref. [17]), and works very well if the centroid points are well separated and are close enough to their respective characters. We are now ready to propose an alternative circuit to the quatum circuit of SVM.

To do so, we use transformations that transfer the centriod points of the classes $A$ and $B$ to the equator cicle

$$
\left(x_{1}^{*}, x_{2}^{*}\right) \stackrel{\text { proj. }}{\longrightarrow}\left(r_{1}^{*}, r_{2}^{*}, r_{3}^{*}\right) \stackrel{\text { rot. }}{\longrightarrow}\left(\bar{r}_{1}^{*}, \bar{r}_{2}^{*}\right),
$$

where $\left(x_{1}^{*}, x_{2}^{*}\right)$ is the component of the centroid point. The projection in 9 is the stereographic projection that projects the centroid point on the Bloch sphere. Then we apply a rotation. This rotation is defined as follows. Let introduce two vectors $\vec{n}$ and $\vec{m}$. The first vector is the cross product of the two centroid vectors $\vec{n}=\left(n_{1}, n_{2}, n_{3}\right):=\overrightarrow{r_{a}^{*}} \times \overrightarrow{r_{b}^{*}}$. The second vector is obtained by rotating the vector $\vec{n}$ about the $Z$ axis by an angle $\phi=\arctan \left(-n_{1} / n_{2}\right)$, i.e., $\vec{m}=$ $\left(m_{1}, m_{2}, m_{3}\right):=R_{Z}(\phi) \vec{n}$. Next we rotate the training points by the rotation $R(\theta, \phi)=R_{X}(\theta) R_{Z}(\phi)$ with $\theta=-\arccos \left(m_{3} /|\vec{m}|\right)$ and $R_{X}$ is the rotation about the $X$ axis. So, the rotation in $(9)$ can be written in matrix form

$R(\theta, \phi)=\left[\begin{array}{ccc}\cos \phi & \sin \phi & 0 \\ -\cos \theta \sin \phi & \cos \theta \cos \phi & \sin \theta \\ \sin \theta \sin \phi & -\sin \theta \cos \phi & \cos \theta\end{array}\right]$.

This rotation given by Eq. (10) leads to $r_{a_{3}}^{*}=r_{b_{3}}^{*}=0$, and therefore, the parameter in Eq. 77 becomes $\tilde{K}=1$. So, the quantum discrimination function Eq. (5) will be reduced simply to the form

$$
\begin{aligned}
f(\vec{r}) & =\mathbf{F} . \mathbf{r}=0 \\
& =\left(\bar{r}_{a_{1}^{*}}-\bar{r}_{b_{1}^{*}}\right) r_{1}+\left(\bar{r}_{a_{2}^{*}}-\bar{r}_{b_{2}^{*}}\right) r_{2}=0 .
\end{aligned}
$$

Thus, from Eq. (11) we obtain the values of $\alpha_{i}$, that is, $\alpha_{1}=1$ and $\alpha_{2}=-1$. This is our main result. Thus, we have found the values of $\alpha_{i}$ without the need of quantum matrix inversion algorithm. 


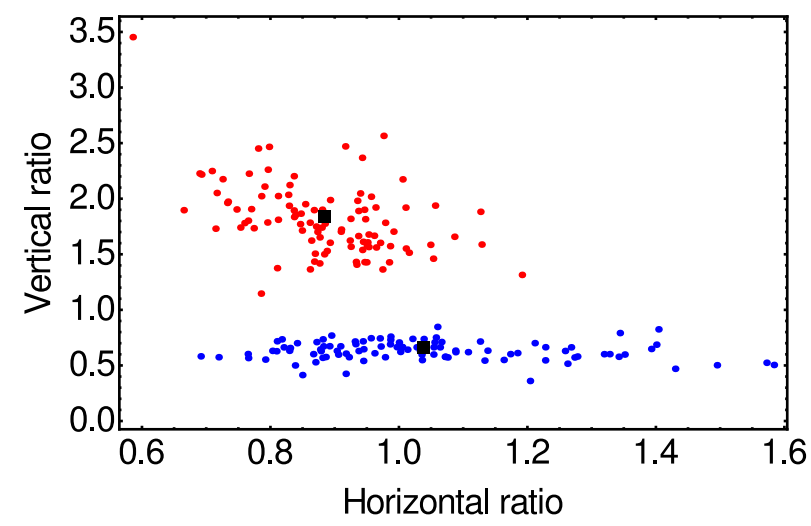

Figure 1: The handwritten characters images from Ref. [22] are given in portable bitmap format. They are cropped and then converted to two-dimentional vectors. The red(blue) color is for the handwritten characters "6(9)". The upper(lower) black square represents the centroid point of the handwritten characters "6(9)".

Let write the centroid points as

$$
\begin{array}{ll}
\left(\bar{r}_{a_{1}^{*}}, \bar{r}_{a_{2}^{*}}\right):=\left(\cos \left(\theta_{1} / 2\right), \sin \left(\theta_{1} / 2\right)\right), & \text { for class } A \\
\left(\bar{r}_{b_{1}^{*}}, \bar{r}_{b_{2}^{*}}\right):=\left(\cos \left(\theta_{2} / 2\right), \sin \left(\theta_{2} / 2\right)\right), & \text { for class } B
\end{array}
$$

These vectors represent the training points for the quantum circuit where $\theta_{i}=\operatorname{arccot}\left[\left(\bar{r}_{i}^{*}\right)_{1} /\left(\bar{r}_{i}^{*}\right)_{2}\right]$.

In general the query vector has three components it is a three-dimensional vector. In order to convert it to two-dimensional vector we can use the following transformation

$$
\left(r_{1}, r_{2}, r_{3}\right) \stackrel{\text { proj. }}{\longrightarrow} \frac{1}{\sqrt{r_{1}^{2}+r_{2}^{2}}}\left(r_{1}, r_{2}\right),
$$

and the query point can also be written in the form

$$
\left(\bar{r}_{1}, \bar{r}_{2}\right):=\left(\cos \left(\theta_{0} / 2\right), \sin \left(\theta_{0} / 2\right)\right) .
$$

Finally, The quantum discriminant function Eq. (11) becomes

$$
f\left(\vec{r}_{0}\right)=\cos \left(\frac{\theta_{1}-\theta_{0}}{2}\right)-\cos \left(\frac{\theta_{2}-\theta_{0}}{2}\right) .
$$

Since we do not need quantum inverse matrix algorithm the implementation of quantum circuit based on NMC can be simplified from QSVM and it is shown in Fig. 2 It consists only of two parts. The first part (dashed) is a training data oracle quantum circuit that is used in Ref. [17] to obtain the kernel matrix up to a constant factor. It consists of two controlled rotation gates about the $y$-axis with two angles depending on the training data and they are given by $\theta_{i}=\operatorname{arccot}\left[\left(r_{i}^{*}\right)_{1} /\left(r_{i}^{*}\right)_{2}\right]$. If the initial state is written as

$$
\left|\psi_{0}\right\rangle=|0\rangle \otimes\left(\frac{|0\rangle-|1\rangle}{\sqrt{2}}\right) \otimes\left(\cos \frac{\theta_{a}}{2}|0\rangle+\sin \frac{\theta_{a}}{2}|1\rangle\right),
$$

where $\theta_{a} \in[0, \pi]$. the state of the system becomes after the oracle quantum circuit

$$
\begin{aligned}
|\psi\rangle= & \frac{1}{\sqrt{2}} \cos \frac{\theta_{a}}{2}(|000\rangle-|010\rangle) \\
& +\frac{1}{\sqrt{2}} \cos \frac{\theta_{1}}{2} \sin \frac{\theta_{a}}{2}|001\rangle \\
& -\frac{1}{\sqrt{2}} \cos \frac{\theta_{2}}{2} \sin \frac{\theta_{a}}{2}|100\rangle \\
& +\frac{1}{\sqrt{2}} \sin \frac{\theta_{1}}{2} \sin \frac{\theta_{a}}{2}|101\rangle \\
& -\frac{1}{\sqrt{2}} \sin \frac{\theta_{2}}{2} \sin \frac{\theta_{a}}{2}|111\rangle .
\end{aligned}
$$

The second part of the quantum circuit (dotted) is also used to implement quantum support vector machine [17] and it is used to introduce the query vector $\overrightarrow{r_{0}}$. It consists of rotation about the $y$-axis and Hadamrd gate. At the output of the simplified quantum circuit the state of the system is given by

$$
\begin{aligned}
|\psi\rangle= & \frac{1}{\sqrt{2}} \cos \frac{\theta_{a}}{2}(|000\rangle-|010\rangle) \\
& +\frac{1}{2}\left(\cos \frac{\theta_{1}-\theta_{0}}{2}-\cos \frac{\theta_{2}-\theta_{0}}{2}\right) \sin \frac{\theta_{a}}{2}|001\rangle \\
& +\frac{1}{2}\left(\cos \frac{\theta_{1}-\theta_{0}}{2}+\cos \frac{\theta_{2}-\theta_{0}}{2}\right) \sin \frac{\theta_{a}}{2}|011\rangle \\
& -\frac{1}{2}\left(\sin \frac{\theta_{1}-\theta_{0}}{2}-\sin \frac{\theta_{2}-\theta_{0}}{2}\right) \sin \frac{\theta_{a}}{2}|101\rangle \\
& -\frac{1}{2}\left(\sin \frac{\theta_{1}-\theta_{0}}{2}+\sin \frac{\theta_{2}-\theta_{0}}{2}\right) \sin \frac{\theta_{a}}{2}|111\rangle
\end{aligned}
$$

One can see from the last equation that the quantum discriminant function appears as coefficient to the 


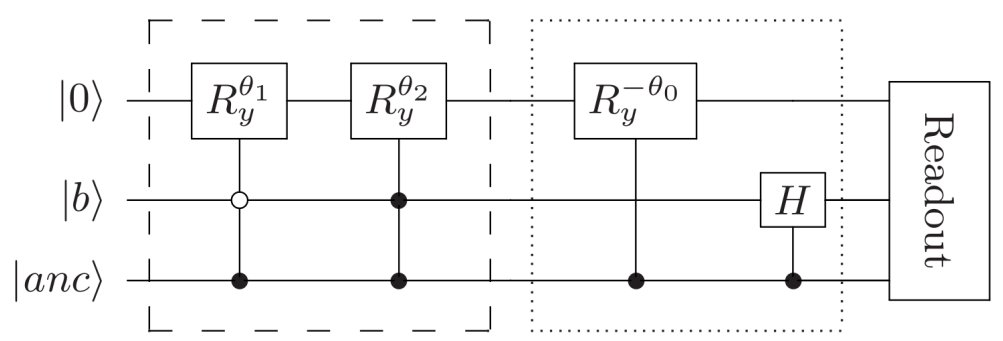

Figure 2: Simplified quantum circuit based on NMC. The state $|b\rangle=(|0\rangle-|1\rangle) / \sqrt{2}$ and $\mid$ anc $\rangle=\cos \theta_{a} / 2|0\rangle+\sin \theta_{a} / 2|1\rangle$. The rotation angles are $\theta_{i}=\operatorname{arccot}\left[\left(r_{i}^{*}\right)_{1} /\left(r_{i}^{*}\right)_{2}\right]$ with $i=0,1,2$.

state $|001\rangle$. So, the expected value of the coherence operator $O=|001\rangle\langle 000|$ which is given by

$$
\langle\psi|O| \psi\rangle=\frac{1}{4 \sqrt{2}} f\left(\vec{r}_{0}\right) \sin \theta_{a},
$$

can be used to classify the query vector. If $\sin \theta_{a}$ is chosen to be positive, the sign of expected value of the coherent operator gives the classification of the query vector.

It is worth mentioning that if there is no ancilla, $\cos \left(\theta_{a} / 2\right)=0$, i.e., $|a n c\rangle=|1\rangle$, we have

$$
\begin{aligned}
|\psi\rangle= & \frac{1}{2}\left(\cos \frac{\theta_{1}-\theta_{0}}{2}-\cos \frac{\theta_{2}-\theta_{0}}{2}\right)|00\rangle \\
& +\frac{1}{2}\left(\cos \frac{\theta_{1}-\theta_{0}}{2}+\cos \frac{\theta_{2}-\theta_{0}}{2}\right)|01\rangle \\
& -\frac{1}{2}\left(\sin \frac{\theta_{1}-\theta_{0}}{2}-\sin \frac{\theta_{2}-\theta_{0}}{2}\right)|10\rangle \\
& -\frac{1}{2}\left(\sin \frac{\theta_{1}-\theta_{0}}{2}+\sin \frac{\theta_{2}-\theta_{0}}{2}\right)|11\rangle .
\end{aligned}
$$

The last equation can be used if the coefficients of $|01\rangle$ does not change its sign. In this case we can use temography to determine the final state Eq. (17), and then classify the query accordingly. In other way, it is important to check the sign of the coefficients $|00\rangle$ and $|01\rangle$. From Fig. 1 we can show that the coefficient of $|01\rangle$ is always positive. In this case we need only two qubits to classify the query, i.e., the ancilla state is not needed in this case.

In the next section we will show explicitly how to apply the transformations and the NMC to classify a query.

\section{Classifying HANDWRITTEN CHARACTERS "6" AND "9"}

In this section we implement the simple quantum circuit for pattern recognition based on NMC for the two handwriting characters " 6 " and "9". We follow
Zhaokai Li et al. [17] and convert each handwriting characters from Ref. [22] to vectors of two dimension. This has been done by considering the horizontal and vertical ratios of these handwriting characters.

Figure 1 shows the horizontal and vertical ratios of the two classes, the handwriting characters " 6 " and " 9 ". One can see that they are linearly separated. Using the stereographic projection describe in section II we map each point on Fig. 1 to a point on the Bloch sphere. This is shown in Fig. 3 The points on the Bloch sphere remain linearly separated. Applying the rotation given by Eq. (10) to all points on the Bloch sphere we obtain the left figure in Fig. 4 This rotation brings the two centroid points on the $x y$-plane which leads to the simplification of the quantum circuit proposed in Ref. [17]. Only the centroid points are transformed to two-dimensional vectors. These points are used as training points. The query point is on the Bloch sphere and it is a three-dimensional vector. One can see that the discriminant function Eq. (11) does not depend on $r_{3}$. So, to convert it to a two-dimensional vector, we apply the projection (12) This transformation is shown in right figure of Fig. 4 and eventually it does not effect the separation of the two classes.

Finally, the training and the query points are now represented by two-dimension vectors. The centroids points for " 6 " and "9" are

$$
\begin{array}{ll}
\text { "6"* }: & (0.884,1.814) \stackrel{\text { rot. and proj. }}{\longrightarrow}(0.583,-0.812) \\
\text { "9"* }: & (1.039,0.631) \stackrel{\text { rot. and proj. }}{\longrightarrow}(0.966,-0.258)
\end{array}
$$

Let for example take the ancilla state to be in the state $\mid$ anc $\rangle=(|0\rangle+|1\rangle) / \sqrt{2}$. If the query chosen is " 6 " (see Fig. 5), the final state would be given by

$$
\begin{aligned}
|\psi\rangle= & 0.653|000\rangle+0.0155|001\rangle-0.653|010\rangle \\
& +0.366|011\rangle-0.0635|101\rangle+0.0895|111\rangle .
\end{aligned}
$$

It is clear that the coefficient of $|001\rangle$ is positive. So, the character represents " 6 ". So, the simple quantum 


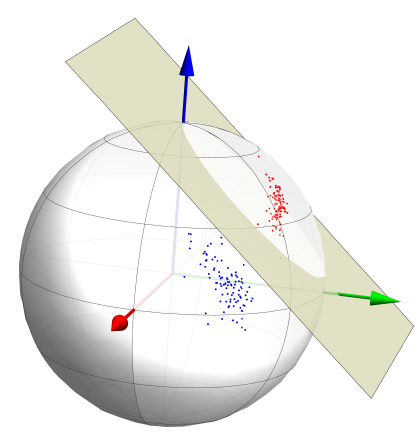

Figure 3: The handwriting characters " 6 " and " 9 " are linearly separated in the Bloch sphere.

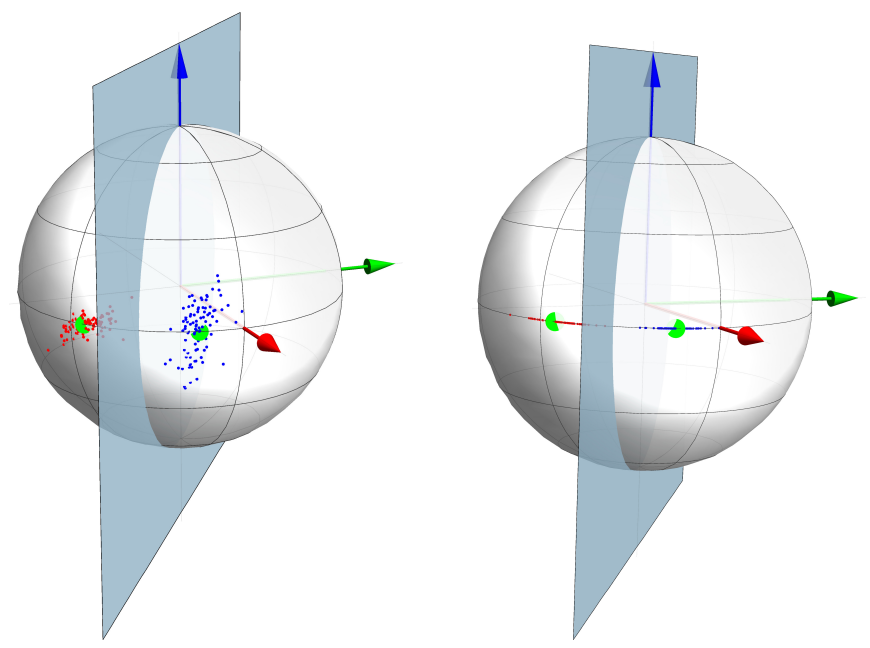

Figure 4: Applying the rotation, Eq. 10, to the Fig. 3, the handwriting characters " 6 " and "9" are linearly separated in the Bloch sphere. The two centroid points are now in the xy-plane (green dots) (left figure). Applying the projection, Eq. 12, to the left figure the handwriting characters are linearly separated and all the points are now in the xy-plane (right figure).

circuit recognizes the handwriting character. Similarly, if we use the query "9" from Fig. 5 we obtain

$$
\begin{aligned}
|\psi\rangle= & 0.653|000\rangle-0.0104|001\rangle-0.653|010\rangle \\
& +0.372|011\rangle-0.0646|101\rangle-0.0602|111\rangle .
\end{aligned}
$$

In this case the coefficient of $|001\rangle$ is negative which indicates that the simple quantum circuit recognizes it as " 9 ".

It is worth mentioning that if the normalized twofeature patterns, $\frac{\vec{x}}{|\vec{x}|}$, are well linearly separated we can dispense the rotation Eq. 10). Thus, the normalized process is enough since the centroid points are now located on the equator circle (see Fig. 6). In this case, the handwriting charachtern " 6 " from Fig 5 is represented by the normalized vector $(0.316,0.949)$ which leads to the final state

$$
\begin{aligned}
|\psi\rangle= & 0.653|000\rangle+0.0113|001\rangle-0.653|010\rangle \\
& +0.371|011\rangle+0.0533|101\rangle-0.0784|111\rangle .
\end{aligned}
$$

The coefficient of $|001\rangle$ is positive. Similarly, for the handwriting character " 9 " represented by the normal-

\begin{tabular}{|c|c|c|}
\hline character & $\left(x_{1}, x_{2}\right)$ & $\left(\bar{x}_{1}, \bar{x}_{2}\right)$ \\
\hline $\mathbf{6}$ & $(0.725,2.175)$ & $(0.468,-0.884)$ \\
\hline $\mathbf{9}$ & $(1.027,0.663)$ & $(0.960,-0.280)$ \\
\hline
\end{tabular}

Figure 5: The two characters used for pattern recognition taken from Ref. [22]. The vector $\left(x_{1}, x_{2}\right)$ represents the two-feature, $x_{1}$ is the horizontal ratio and $x_{2}$ is the vertical ratio of the pixels of the image. the vector $\left(\bar{x}_{1}, \bar{x}_{2}\right)$ is obtained by using the rotation and the projection defined in the text. 


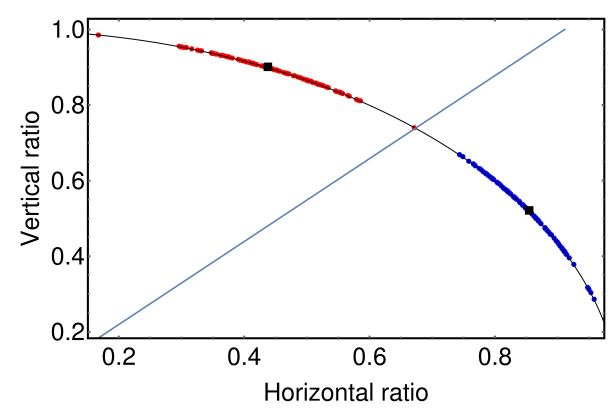

Figure 6: The handwriting characters are still linearly separated after normalization. The equation of line is given by the quantum discriminant function $\mathrm{Eq}$. 111.

ized vector $(0.907,0.422)$ leads to the final state

$$
\begin{aligned}
|\psi\rangle= & 0.653|000\rangle-0.007|001\rangle-0.653|010\rangle \\
& +0.376|011\rangle+0.0541|101\rangle+0.0488|111\rangle,
\end{aligned}
$$

and the coefficient in this case is negative. Therefore, if the two-feature patterns represented by normalized vectors are linearly separated we can skip the rotation and projection. The quantum discrimination function is already in its simplest form given by Eq. (11) where the values of $\alpha_{i}$ are \pm 1 .

We have proposed a simplified quantum circuit based on pattern recognition on the quantum Bloch sphere and the nearest mean classifier (NMC) to classify the handwritting characters. This quantum circuit does not need quantum matrix inversion and can be implemented by using three-qubit NMR processor. The hyperplane can be found using projection and rotation on the Bloch sphere. The rotation $R(\theta, \phi)$ transforms the two centroid points to the equator circle, so that the values of $\alpha_{i}$ are \pm 1 . As a result of this, the quantum matrix inversion is not needed. The projection is used to transform the query from three-dimention to two-dimention since the quantum discrimination function does not depend on the third component of the query vector. It is also worth to mention that if the normalized two-feature patterns are linearly separable then the rotation of the training data are not needed. In some cases we can extend two-feature patterns to a three-feature patterns if the normalized three-feature patterns can leads to linearly separated classes.

\section{REFERENCES}

[1] P. Wittek, Quantum machine learning: what quantum computing means to data mining (Academic Press, 2014).

[2] M. Schuld, I. Sinayskiy, and F. Petruccione, Quantum computing for pattern classification, in Pacific
Rim International Conference on Artificial Intelligence, pp. 208-220, Springer, 2014.

[3] M. Schuld, I. Sinayskiy, and F. Petruccione, Contemporary Physics 56, 172 (2015).

[4] N. Wiebe, A. Kapoor, and K. Svore, arXiv preprint arXiv:1401.2142 (2014).

[5] T. Cover and P. Hart, IEEE transactions on information theory 13, 21 (1967).

[6] N. M. Ball and R. J. Brunner, International Journal of Modern Physics D 19, 1049 (2010).

[7] R. O. Duda, P. E. Hart, and D. G. Stork, Pattern classification (John Wiley \& Sons, 2012).

[8] A. R. Webb, Statistical pattern recognition (John Wiley \& Sons, 2003).

[9] C. Bishop, Pattern Recognition and Machine Learning (Information Science and Statistics), 1st edn. 2006. corr. 2nd printing edn (Springer, New York, 2007).

[10] R. S. Michalski, J. G. Carbonell, and T. M. Mitchell, Machine learning: An artificial intelligence approach (Springer Science \& Business Media, 2013).

[11] K. P. Murphy, Machine learning: a probabilistic perspective (MIT press, 2012).

[12] P. Rebentrost, M. Mohseni, and S. Lloyd, Phys. Rev. Lett. 113, 130503 (2014).

[13] A. W. Harrow, A. Hassidim, and S. Lloyd, Phys. Rev. Lett. 103, 150502 (2009).

[14] N. Wiebe, D. Braun, and S. Lloyd, Phys. Rev. Lett. 109, 050505 (2012).

[15] Y. Cao, A. Daskin, S. Frankel, and S. Kais, Molecular Physics 110, 1675 (2012).

[16] J. Pan et al., Phys. Rev. A 89, 022313 (2014). 
[17] Z. Li, X. Liu, N. Xu, and J. Du, Phys. Rev. Lett. 114, 140504 (2015).

[18] N. Wiebe, A. Kapoor, and K. Svore, arXiv preprint arXiv:1401.2142 (2014).

[19] G. Sergioli, E. Santucci, L. Didaci, J. A. Miskczak, and R. Giuntini, arXiv preprint arXiv:1603.00173 (2016).
[20] H. S. M. Coxeter, Introduction to geometry (New York, London, 1961).

[21] J. Friedman, T. Hastie, and R. TibshiraniThe elements of statistical learning Vol. 1 (Springer series in statistics Springer, Berlin, 2001).

[22] The handwitten characters are taken from https://github.com/damiles/basicOCR. 